\title{
Usefulness of contralateral carotid angiography in revascularization therapy of acute internal carotid artery occlusion
}

\author{
Kiyoshi Tsuji, MD, PhD, ${ }^{1}$ Nobuhiro Nakagawa, MD, PhD, ${ }^{1}$ Norihito Fukawa, MD, ${ }^{1}$ \\ Masaharu Miyauchi, MD, ${ }^{1}$ Kentaro Furukawa, MD, ${ }^{1}$ Kinya Nakanishi, MD, PhD, ${ }^{2}$ \\ Naoki Nakano, MD, PhD, ${ }^{1}$ Kazuo Kataoka, MD, PhD, ${ }^{2}$ and Amami Kato, MD, PhD ${ }^{1}$ \\ 'Department of Neurosurgery, Kindai University Faculty of Medicine, Osaka; and 'Department of Neurosurgery, Nara Hospital, \\ Kindai University Faculty of Medicine, Nara, Japan
}

\begin{abstract}
OBJECTIVE The vascular lumen of an acutely occluded internal carotid artery (ICA) generally contains numerous thrombi. Therefore, carotid angiography on the affected side during revascularization therapy of acute ICA occlusion has a potential risk of causing distal embolization. In this study the authors propose the use of contralateral carotid angiography.
\end{abstract}

METHODS Six patients with acute ICA occlusion underwent revascularization therapy using a stent retriever or Penumbra system. Revascularization therapy was performed with placement of a 9-Fr balloon-guiding catheter (BGC) in the affected ICA and a 4-Fr diagnostic catheter in the contralateral ICA. During the procedure, the 9-Fr BGC was kept inflated, and all control angiography was performed from the 4-Fr diagnostic catheter. After thrombectomy, contralateral carotid angiography combined with manual aspiration from the 9-Fr BGC was performed to assess the presence or absence of residual thrombi in the affected ICA. The 9-Fr BGC was deflated only after the complete absence of residual thrombi in the affected ICA was confirmed.

RESULTS The time required for introducing the 4-Fr diagnostic catheter into the contralateral ICA was within a few minutes in all patients. Residual thrombi in the affected ICA were found in 3 of 6 patients. The residual thrombi in these 3 patients were completely removed; thus, distal embolization was prevented.

CONCLUSIONS Contralateral carotid angiography is useful for avoiding distal embolization during revascularization therapy of acute ICA occlusion. Further studies involving a larger number of patients are warranted to verify the clinical efficacy of this contralateral carotid angiography.

https://thejns.org/doi/abs/10.3171/2017.3.JNS162563

KEY WORDS acute internal carotid artery occlusion; balloon-guiding catheter; contralateral carotid angiography; revascularization therapy; stent retriever; thrombectomy; vascular disorders

I SCHEMIC stroke caused by acute internal carotid artery (ICA) occlusion is often associated with poor outcomes. ${ }^{10,13}$ Various revascularization therapies were previously used to reopen an acutely occluded ICA, but satisfactory treatment outcomes were not achieved. ${ }^{2,5,7,9}$ Most recently, several clinical studies have shown that revascularization therapy using a stent retriever can improve clinical outcomes in patients with acute ICA occlusion. .,6,12 $^{1}$
The vascular lumen of an acutely occluded ICA generally contains numerous thrombi. ${ }^{7,9,10}$ Therefore, because a single thrombectomy attempt with a stent retriever may not completely remove all thrombi, carotid angiography on the affected side to confirm recanalization has a potential risk of causing distal embolization. In this report, the usefulness of contralateral carotid angiography in revascularization therapy of acute ICA occlusion is described.

ABBREVIATIONS ACOA = anterior communicating artery; AP = anteroposterior; ASPECTS = Alberta Stroke Program Early CT Score; BGC = balloon-guiding catheter; DWI = diffusion-weighted imaging; ICA = internal carotid artery; MCA = middle cerebral artery; MRA = MR angiography; NIHSS = National Institutes of Health Stroke Scale; TICI $=$ Thrombolysis in Cerebral Infarction; tPA = tissue plasminogen activator.

SUBMITTED October 8, 2016. ACCEPTED March 10, 2017.

INCLUDE WHEN CITING Published online September 15, 2017; DOI: 10.3171/2017.3.JNS162563. 
TABLE 1. Clinical data of 6 patients with acute ICA occlusion

\begin{tabular}{|c|c|c|c|c|c|c|c|c|c|}
\hline $\begin{array}{l}\text { Case } \\
\text { No. }\end{array}$ & $\begin{array}{l}\text { Age (yrs), } \\
\text { Sex }\end{array}$ & $\begin{array}{l}\text { Stroke } \\
\text { Etiology }\end{array}$ & $\begin{array}{l}\text { Admission } \\
\text { NIHSS Score }\end{array}$ & $\begin{array}{l}\text { Intravenous } \\
\text { tPA }\end{array}$ & $\begin{array}{l}\text { Site of Diagnostic } \\
\text { Catheter Placement }\end{array}$ & $\begin{array}{l}\text { Residual } \\
\text { Thrombi }\end{array}$ & $\begin{array}{l}\text { Final TICl } \\
\text { Grade }\end{array}$ & Complications & $\begin{array}{l}\text { Discharge } \\
\text { NIHSS Score }\end{array}$ \\
\hline 1 & $94, F$ & $\begin{array}{r}\text { Cardiogenic } \\
\text { embolism }\end{array}$ & 16 & No & Contralateral ICA & No & 3 & $\begin{array}{l}\text { ICA dissection on } \\
\text { the affected side }\end{array}$ & 14 \\
\hline 2 & $67, \mathrm{~F}$ & $\begin{array}{r}\text { Cardiogenic } \\
\text { embolism }\end{array}$ & 11 & No & Contralateral ICA & Yes & 3 & None & 2 \\
\hline 3 & $69, \mathrm{M}$ & $\begin{array}{r}\text { Cardiogenic } \\
\text { embolism }\end{array}$ & 31 & Yes & Vertebral artery & Yes & 3 & None & Deceased \\
\hline 4 & $33, F$ & Dissection & 16 & Yes & Contralateral ICA & No & $2 b$ & None & 5 \\
\hline 5 & $38, F$ & $\begin{array}{r}\text { Paradoxical } \\
\text { embolism }\end{array}$ & 14 & No & Contralateral ICA & Yes & $2 b$ & None & 10 \\
\hline 6 & $79, \mathrm{M}$ & Arteriosclerosis & 10 & No & Contralateral ICA & No & $2 b$ & None & 4 \\
\hline
\end{tabular}

\section{Methods}

Patients

Six patients ( 2 men and 4 women, mean age 63 years) with acute ICA occlusion underwent revascularization therapy (Table 1). The etiology of acute ICA occlusion in these 6 patients was cardiogenic embolism $(n=3)$, dissection $(\mathrm{n}=1)$, paradoxical embolism $(\mathrm{n}=1)$, and arteriosclerosis $(\mathrm{n}=1)$. The mean National Institutes of Health Stroke Scale (NIHSS) score on admission was 16 (range 10-31). Intravenous tissue plasminogen activator (tPA) was administered to 2 patients who met the indications (Table 1). Written informed consent was obtained from all patients.

\section{Procedure}

Revascularization therapy was performed under local anesthesia in all patients. A 9-Fr sheath was placed in the right femoral artery, and a 4-Fr sheath was placed in the left femoral artery. First, diagnostic angiography was performed to evaluate the occlusion site and collateral flow. After diagnostic angiography, a 9-Fr balloon-guiding catheter (BGC) was placed in the affected ICA, and a 4-Fr diagnostic catheter was placed in the contralateral ICA; in Case 3, the 4-Fr diagnostic catheter was placed in the vertebral artery because the anterior communicating artery (ACoA) was hypoplastic and the posterior communicating artery was patent. During the procedure, the 9-Fr BGC was kept inflated. All control angiography was performed from the 4-Fr diagnostic catheter. A stent retriever or Penumbra system (Penumbra) was used for thrombectomy. After thrombectomy, contralateral carotid angiography combined with manual aspiration from the 9-Fr BGC was performed to assess the presence or absence of residual thrombi in the affected ICA; in Case 3, vertebral angiography combined with manual aspiration from the 9-Fr BGC was performed. This contralateral carotid angiography or vertebral angiography was performed by manual injection instead of using a power injector. A first operator performed manual injection using a 10-ml syringe, and a second operator performed manual aspiration from the $9-\mathrm{Fr}$ BGC using a $20-\mathrm{ml}$ syringe. The 9-Fr BGC was deflated only after the complete absence of residual thrombi in the affected ICA was confirmed.

\section{Results}

The time required for introducing the 4-Fr diagnostic catheter into the contralateral ICA was within a few minutes in all patients. Residual thrombi in the affected ICA were found in 3 of 6 patients. The residual thrombi in these 3 patients were completely removed, and therefore distal embolization was prevented. The final Thrombolysis in Cerebral Infarction (TICI) grade was 3 in 3 of the 6 patients and $2 \mathrm{~b}$ in the remaining 3 patients. One complication was encountered, in the form of ICA dissection on the affected side when advancing the 9-Fr BGC (Case 1). One patient (Case 3) died of severe brain edema after cerebral infarction (Table 1).

\section{Illustrative Cases \\ Case 2}

A 67-year-old woman with a history of chronic atrial fibrillation developed sudden left hemiparesis and slurred speech, and she was transported by ambulance to our hospital 150 minutes after symptom onset. Her NIHSS score on admission was 11. Head CT showed no obvious abnormalities. Magnetic resonance diffusion-weighted imaging (DWI) showed a small acute infarction in the right frontal lobe (DWI-Alberta Stroke Program Early CT Score $[$ ASPECTS] = 9; Fig. 1 left), and MR angiography (MRA) showed right ICA occlusion (Fig. 1 right). Since her prothrombin time/international normalized ratio was prolonged to 1.95 because of warfarin therapy, intravenous tPA was not administered.

Under local anesthesia, a 9-Fr sheath was placed in the right femoral artery, and a 4-Fr sheath was placed in the left femoral artery. After sheath placement, 5000 units of heparin were administered intravenously. First, diagnostic angiography was performed using a 4-Fr diagnostic catheter. Right common carotid angiography showed occlusion of the right ICA (Fig. 2A). Left internal carotid angiography showed that the right carotid terminus was occluded by a thrombus (Fig. 2B). After diagnostic angiography, the 4-Fr diagnostic catheter was kept in the left ICA instead of being removed, and an Optimo 9-Fr BGC (Tokai Medical Products) was placed in the right ICA. During the procedure, the 9-Fr BGC was kept in- 

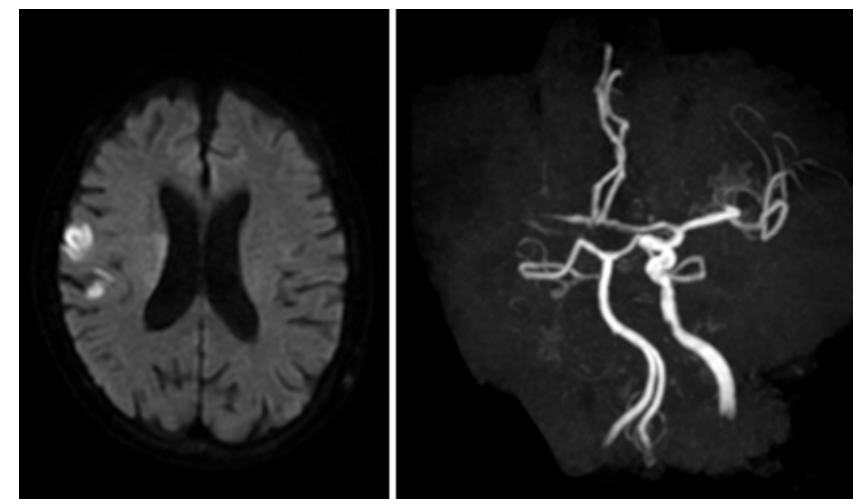

FIG. 1. Case 2. MR-DWI (left) shows a small acute infarction in the right frontal lobe. MRA (right) shows right ICA occlusion.

flated. All control angiography was performed from the 4-Fr diagnostic catheter. By use of a standard technique, a Trevo Pro 18 microcatheter (Stryker Neurovascular) was navigated distal to the thrombus over a CHIKAI 0.014inch microguidewire (Asahi Intecc). A Trevo ProVue $4 \times$ $20-\mathrm{mm}$ stent retriever (Stryker Neurovascular) was deployed over the entire length of the thrombus. Left internal carotid angiography after stent deployment confirmed immediate flow restoration (Fig. 2C). After 5 minutes, the stent was gently withdrawn under manual aspiration through the BGC. A large thrombus was removed with the first attempt, and left internal carotid angiography showed recanalization of the right carotid terminus (Fig. 2D). However, backflow of blood from the proximal end of the BGC was not observed, and blood aspiration was not possible. Some residual thrombus was therefore believed to be present. Because the right cerebral hemisphere was being perfused with cross-flow via the ACoA, the search for the residual thrombi was performed slowly, without haste. A residual thrombus was found just distal to the BGC tip. This residual thrombus was aspirated using a Penumbra 5MAX ACE reperfusion catheter (Penumbra). Thereafter, backflow of blood was observed from the proximal end of the BGC. To assess whether further residual thrombi were present, contralateral carotid angiography combined with manual aspiration from the BGC was performed (Fig. 2E). After the complete absence of residual thrombi in the affected ICA was confirmed, the BGC was deflated for the first time. The final TICI grade was 3 . Final right internal carotid angiography showed vasospasm in the right petrous ICA attributable to BGC placement (Fig. 2F). It was believed that the residual thrombus had likely remained after being caught at this site of vasospasm.

The patient's neurological symptoms improved dramatically after the procedure, and she became ambulatory. Her NIHSS score at the time of discharge was 2.

\section{Case 5}

A 38-year-old woman had been admitted to the mater-
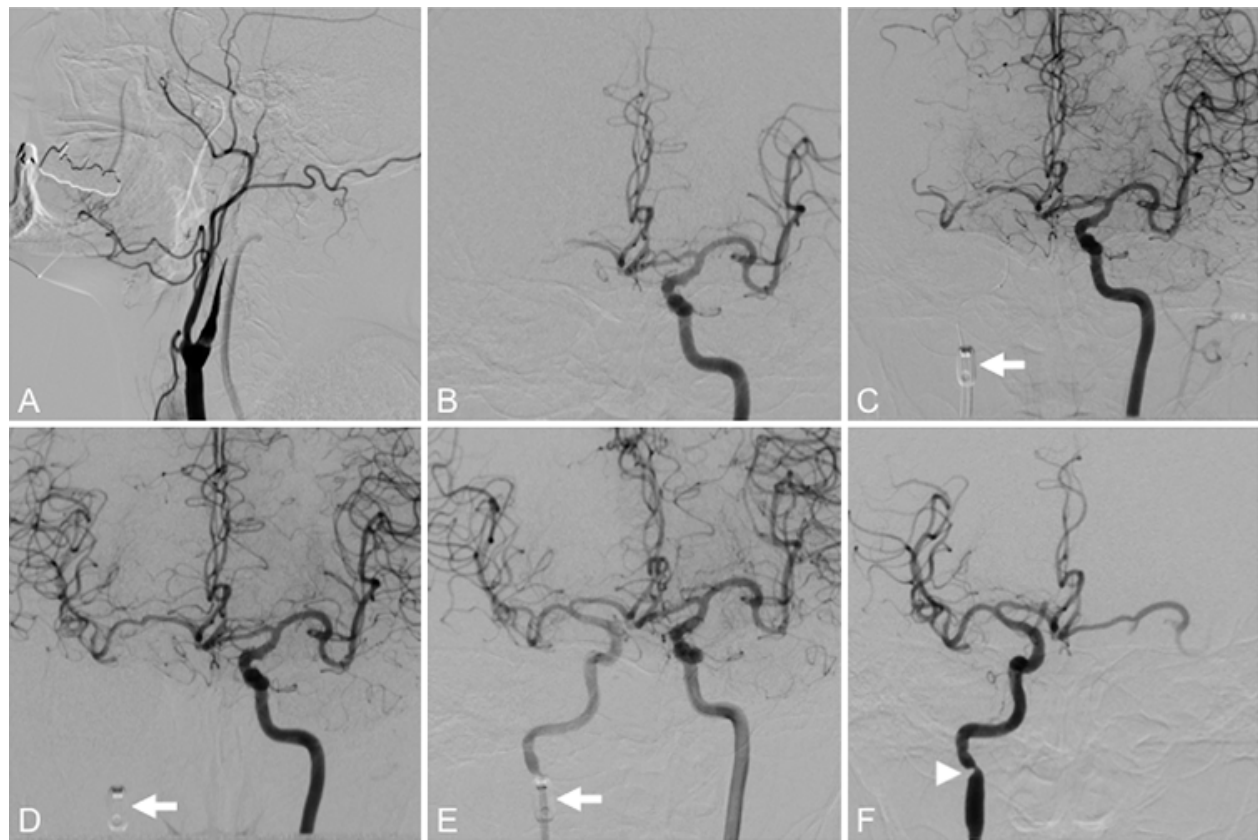

FIG. 2. Case 2. A: Right common carotid angiography (lateral view) shows occlusion of the right ICA. B: Left internal carotid angiography (anteroposterior [AP] view) shows that the right carotid terminus is occluded by a thrombus. C: Left internal carotid angiography (AP view) after Trevo ProVue stent placement shows immediate flow restoration. The arrow shows the inflated balloon of the BGC. D: Left internal carotid angiography (AP view) after thrombus removal shows recanalization of the right carotid terminus. The arrow shows the inflated balloon of the BGC. E: Left internal carotid angiography combined with manual aspiration from the BGC (AP view) confirms the absence of residual thrombi in the affected right ICA. The arrow shows the inflated balloon of the BGC. F: Right internal carotid angiography (AP view) after the procedure shows TICI Grade 3 flow of the right ICA. The arrowhead shows vasospasm in the right petrous ICA, believed to be due to BGC placement. 
nity ward at our hospital for delivery. Two days after delivery, she developed sudden disturbance of consciousness and left hemiparesis, and she was referred to our department. Her NIHSS score was 14 at symptom onset. Head CT showed no obvious hemorrhagic lesions. MR-DWI showed faint high signal intensity in the right basal ganglia (DWI-ASPECTS = 7; Fig. 3 left), and MRA showed right ICA occlusion (Fig. 3 right). Because the patient had given birth 2 days earlier, intravenous tPA was not administered. Endovascular therapy was started 170 minutes after symptom onset.

Under local anesthesia, a 9-Fr sheath was placed in the right femoral artery, and a 4-Fr sheath was placed in the left femoral artery. Six thousand units of heparin were administered intravenously, and then diagnostic angiography was performed using a 4-Fr diagnostic catheter. Right common carotid angiography showed occlusion of the right ICA (Fig. 4A). Left internal carotid angiography showed that the right carotid terminus was occluded by a thrombus (Fig. 4B). The late phase of left internal carotid angiography showed that the right middle cerebral artery (MCA) territory was being perfused with abundant leptomeningeal collateral flow from the right anterior cerebral artery. After diagnostic angiography, the 4-Fr diagnostic catheter was kept in the left ICA instead of being removed, and an Optimo 9-Fr BGC was placed in the right ICA. During the procedure, the 9-Fr BGC was kept inflated. First, manual aspiration from the inflated BGC was performed. Numerous thrombi were aspirated, and blood flowed back from the proximal end of the BGC. Contralateral carotid angiography combined with manual aspiration from the BGC showed recanalization of the right ICA, but the right MCA remained occluded. Furthermore, findings suspicious for a thrombus were observed in the right carotid siphon (Fig. 4C). The thrombus in the right carotid siphon was more clearly visualized on a right anterior oblique view (Fig. 4D). After a Trevo Pro 18 microcatheter was introduced distal to the occlusion site of the right MCA using a CHIKAI 0.014-inch microguidewire, a Trevo ProVue $4 \times 20-\mathrm{mm}$ stent retriever was deployed spanning from the $\mathrm{M}_{1}$ segment of the right MCA to the right carotid siphon. The stent was left in position for 5 minutes and then gently withdrawn under manual aspiration through the BGC. A large thrombus was removed with the stent (Fig. 4E). Contralateral carotid angiography combined with manual aspiration from the BGC showed disappearance of the thrombus in the right carotid siphon, but the right MCA was still occluded. A second attempt with the Trevo ProVue was performed for the right MCA occlusion, but because recanalization was not achieved, the strategy was switched to aspiration thrombectomy with a Penumbra system. A thrombus was aspirated with a Penumbra 5MAX ACE reperfusion catheter, and the right MCA was successfully recanalized. After the complete absence of residual thrombi in the affected ICA was confirmed on contralateral carotid angiography combined with manual aspiration from the BGC (Fig. 4F), the BGC was deflated for the first time. The final TICI grade was $2 b$.

The patient's neurological symptoms improved gradually after the procedure. She was transferred to a rehabilitation hospital 1 month after stroke onset with an
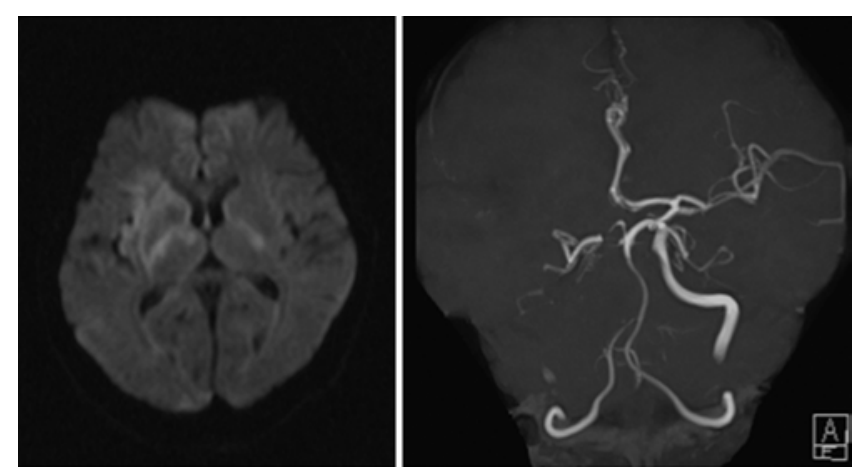

FIG. 3. Case 5. MR-DWI (left) shows faint high signal intensity in the right basal ganglia. MRA (right) shows right ICA occlusion.

NIHSS score of 10 . The cause of cerebral infarction in this patient was paradoxical embolism due to deep venous thrombosis.

\section{Discussion}

The advent of stent retrievers has dramatically improved the treatment outcomes of endovascular therapy for acute ischemic stroke. Several recent randomized controlled trials have shown that revascularization therapy using a stent retriever is effective for acute intracranial large artery occlusion of the anterior circulation, including the ICA. ${ }^{1,6,12}$ Based on these results, the American Heart Association/American Stroke Association guidelines now recommend performing revascularization therapy with a stent retriever in addition to intravenous tPA for eligible patients. ${ }^{11}$ However, revascularization therapy for acute ICA occlusion, which is often associated with numerous thrombi, is not always simple. ${ }^{7,9,10}$

Use of a BGC has been reported to prevent distal embolization during revascularization therapy for acute ischemic stroke. ${ }^{3,4}$ However, in the case of acute ICA occlusion with numerous thrombi, because a single thrombectomy attempt may not completely remove all thrombi, distal embolization may occur when the BGC is deflated. Therefore, in the present case series, we kept the BGC inflated until the absence of residual thrombi in the affected ICA was confirmed. This prevented distal embolization of residual thrombi in 3 of the 6 patients. The ordinary use of a BGC alone may be insufficient to prevent distal embolization during revascularization therapy of acute ICA occlusion. The method described here, namely the combined use of a continuously inflated BGC and contralateral carotid angiography, may provide superior revascularization results in acute ICA occlusion.

Our treatment strategy in revascularization therapy of acute ICA occlusion is to give top priority to removal of the thrombus in the carotid terminus. This is our strategy because removal of a thrombus in the carotid terminus allows collateral flow from the contralateral side. As long as the carotid terminus is patent, there is no need for haste in removing thrombi proximal to the carotid terminus. In the present case series, we first performed contralateral carotid angiography to assess whether the carotid terminus was occluded. If the carotid terminus was occluded, 


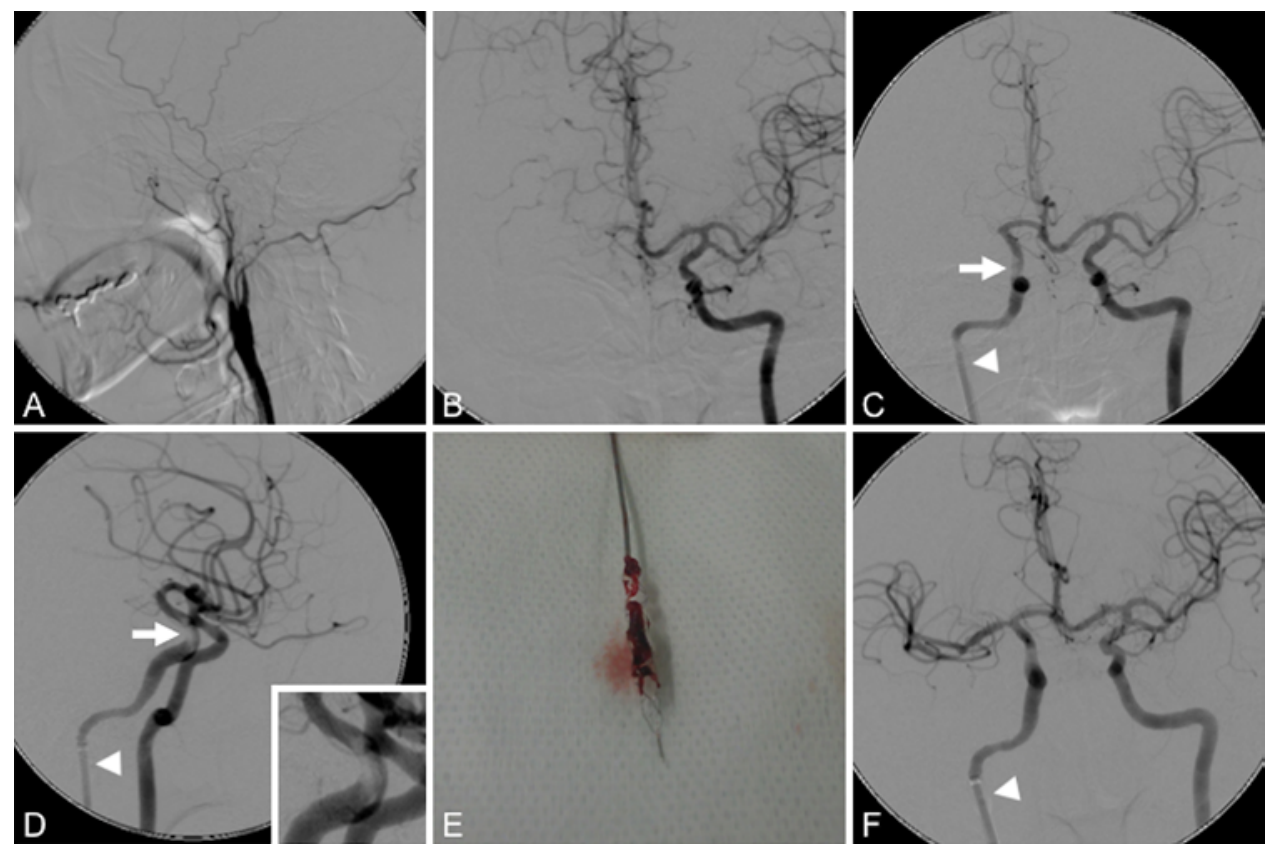

FIG. 4. Case 5. A: Right common carotid angiography (lateral view) shows occlusion of the right ICA. B: Left internal carotid angiography (AP view) shows that the right carotid terminus is occluded by a thrombus. C: Left internal carotid angiography combined with manual aspiration from the BGC (AP view) shows recanalization of the right ICA and the still-occluded right MCA. The arrow shows a suspected thrombus in the right carotid siphon. The arrowhead shows the inflated balloon of the BGC. D: Left internal carotid angiography combined with manual aspiration from the BGC (right anterior oblique view) clearly shows a thrombus (arrow) in the right carotid siphon. The arrowhead shows the inflated balloon of the BGC. The inset is an enlarged view of the thrombus in the right carotid siphon. E: Photograph shows a large thrombus removed with a Trevo ProVue stent. F: Left internal carotid angiography combined with manual aspiration from the BGC (AP view) confirms the absence of residual thrombi in the affected right ICA. The arrowhead shows the inflated balloon of the BGC.

we removed the thrombus in the carotid terminus with top priority, as if to pull it down proximally. Evaluation of whether the carotid terminus had been recanalized after thrombus removal was performed by contralateral carotid angiography. Contralateral carotid angiography, which provides important information such as the occlusion site and collateral circulation during the procedure, was very useful for determining the treatment strategy of acute ICA occlusion.

Even after a thrombus in the carotid terminus is removed, cases in which blood aspiration from the BGC is not possible are occasionally encountered. In such cases, we first recommend performing microcatheter angiography to investigate the cause. Possible causes of the inability to aspirate blood from the BGC besides residual thrombus as described in Case 2 include vasospasm due to BGC placement and severe stenosis due to arteriosclerosis or dissection. If severe stenosis due to arteriosclerosis or dissection is found by microcatheter angiography, the BGC can be temporarily deflated to treat these lesions; however, it is very important that the absence of residual thrombi be confirmed before the BGC is deflated.

We do not recommend the method described herenamely, the combined use of a continuously inflated BGC and contralateral carotid angiography-for revascularization therapy of acute MCA occlusions. The first reason for this is that the thrombus burden in acute MCA occlusion is not as high as that in acute ICA occlusion. The second reason is that prolonged inflation of the BGC can reduce leptomeningeal collateral flow from the ipsilateral anterior cerebral artery, which may further worsen cerebral ischemia in the MCA territory. In Case 5, the BGC was kept inflated until the end of the procedure, but it should have been temporarily deflated when the residual thrombus in the right carotid siphon was completely removed and the condition became right MCA occlusion.

This is the first report to describe the usefulness of contralateral carotid angiography in revascularization therapy of acute ICA occlusion. In situations in which prompt recanalization is desired, introduction of a diagnostic catheter into the contralateral ICA certainly leads to some time loss, but introducing a diagnostic catheter should not take that much time for a neurointerventionalist. ${ }^{8}$ Indeed, in the present case series, the time required for introducing the diagnostic catheter was within a few minutes in all patients. We believe that the benefits provided by contralateral carotid angiography outweigh this time loss of only a few minutes.

\section{Limitations of the Study}

The present study was a case series with a small number of patients, and no comparison with a control group was made. Therefore, we cannot affirm the clinical efficacy of contralateral carotid angiography. To use contralateral carotid angiography, a sufficiently large ACoA and bilateral $\mathrm{A}_{1}$ segments are required. Preoperative assess- 
ment with MRA or CT angiography source images would help determine whether contralateral carotid angiography is appropriate.

\section{Conclusions}

In the present case series, contralateral carotid angiography was very useful for revascularization therapy of acute ICA occlusion because it not only provided important information such as collateral circulation during the procedure but also avoided distal embolization. We hope that the clinical efficacy of this contralateral carotid angiography will be verified in the near future by prospective studies involving a larger number of patients.

\section{References}

1. Berkhemer OA, Fransen PS, Beumer D, van den Berg LA, Lingsma HF, Yoo AJ, et al: A randomized trial of intraarterial treatment for acute ischemic stroke. $\mathbf{N}$ Engl J Med 372:11-20, 2015

2. Broderick JP, Palesch YY, Demchuk AM, Yeatts SD, Khatri P, Hill MD, et al: Endovascular therapy after intravenous t-PA versus t-PA alone for stroke. N Engl J Med 368:893903, 2013

3. Chueh JY, Kühn AL, Puri AS, Wilson SD, Wakhloo AK, Gounis MJ: Reduction in distal emboli with proximal flow control during mechanical thrombectomy: a quantitative in vitro study. Stroke 44:1396-1401, 2013

4. Chueh JY, Puri AS, Wakhloo AK, Gounis MJ: Risk of distal embolization with stent retriever thrombectomy and ADAPT. J Neurointerv Surg 8:197-202, 2016

5. Flint AC, Duckwiler GR, Budzik RF, Liebeskind DS, Smith WS: Mechanical thrombectomy of intracranial internal carotid occlusion: pooled results of the MERCI and Multi MERCI Part I trials. Stroke 38:1274-1280, 2007

6. Goyal M, Demchuk AM, Menon BK, Eesa M, Rempel JL, Thornton J, et al: Randomized assessment of rapid endovascular treatment of ischemic stroke. N Engl J Med 372:10191030,2015

7. Jansen O, von Kummer R, Forsting M, Hacke W, Sartor K: Thrombolytic therapy in acute occlusion of the intracranial internal carotid artery bifurcation. AJNR Am J Neuroradiol 16:1977-1986, 1995

8. Liebeskind DS, Flint AC, Budzik RF, Xiang B, Smith WS, Duckwiler GR, et al: Carotid I's, L's and T's: collaterals shape the outcome of intracranial carotid occlusion in acute ischemic stroke. J Neurointerv Surg 7:402-407, 2015

9. Lin R, Vora N, Zaidi S, Aleu A, Jankowitz B, Thomas A, et al: Mechanical approaches combined with intra-arterial pharmacological therapy are associated with higher recanalization rates than either intervention alone in revascularization of acute carotid terminus occlusion. Stroke 40:2092-2097, 2009

10. Paciaroni M, Balucani C, Agnelli G, Caso V, Silvestrelli G, Grotta JC, et al: Systemic thrombolysis in patients with acute ischemic stroke and internal carotid artery occlusion: the ICARO study. Stroke 43:125-130, 2012

11. Powers WJ, Derdeyn CP, Biller J, Coffey CS, Hoh BL, Jauch EC, et al: 2015 American Heart Association/American Stroke Association Focused Update of the 2013 Guidelines for the Early Management of Patients With Acute Ischemic Stroke Regarding Endovascular Treatment: a guideline for healthcare professionals from the American Heart Association/ American Stroke Association. Stroke 46:3020-3035, 2015

12. Saver JL, Goyal M, Bonafe A, Diener HC, Levy EI, Pereira VM, et al: Stent-retriever thrombectomy after intravenous t-PA vs. t-PA alone in stroke. N Engl J Med 372:2285-2295, 2015

13. Smith WS, Lev MH, English JD, Camargo EC, Chou M, Johnston SC, et al: Significance of large vessel intracranial occlusion causing acute ischemic stroke and TIA. Stroke 40:3834-3840, 2009

\section{Disclosures}

The authors report no conflict of interest concerning the materials or methods used in this study or the findings specified in this paper.

\section{Author Contributions}

Conception and design: Tsuji. Acquisition of data: Tsuji, Nakagawa, Fukawa, Miyauchi, Furukawa, Nakanishi. Analysis and interpretation of data: Tsuji, Nakano. Drafting the article: Tsuji. Critically revising the article: Tsuji. Reviewed submitted version of manuscript: all authors. Approved the final version of the manuscript on behalf of all authors: Tsuji. Study supervision: Kataoka, Kato.

\section{Correspondence}

Kiyoshi Tsuji, Department of Neurosurgery, Kindai University Faculty of Medicine, 377-2 Ohno-Higashi, Osaka-Sayama, Osaka 589-8511, Japan. email: kiyoshi-tsuji@med.kindai.ac.jp. 